\title{
CORRECTION
}

\section{Author Correction: 863 genomes reveal the origin and domestication of chicken}

Ming-Shan Wang, Mukesh Thakur (D, Min-Sheng Peng (D), Yu Jiang, Laurent Alain François Frantz (D), Ming Li (D), Jin-Jin Zhang, Sheng Wang, Joris Peters, Newton Otieno Otecko, Chatmongkon Suwannapoom, Xing Guo, Zhu-Qing Zheng, Ali Esmailizadeh iD, Nalini Yasoda Hirimuthugoda, Hidayat Ashari, Sri Suladari, Moch Syamsul Arifin Zein, Szilvia Kusza, Saeed Sohrabi, Hamed Kharrati-Koopaee, Quan-Kuan Shen (D, Lin Zeng, Min-Min Yang, Ya-Jiang Wu, Xing-Yan Yang, Xue-Mei Lu, Xin-Zheng Jia, Qing-Hua Nie, Susan Joy Lamont, Emiliano Lasagna, Simone Ceccobelli, Humpita Gamaralalage Thilini Nisanka Gunwardana, Thilina Madusanka Senasige, Shao-Hong Feng, Jing-Fang Si, Hao Zhang, Jie-Qiong Jin, Ming-Li Li, Yan-Hu Liu, Hong-Man Chen, Cheng Ma, Shan-Shan Dai, Abul Kashem Fazlul Haque Bhuiyan, Muhammad Sajjad Khan, Gamamada Liyanage Lalanie Pradeepa Silva, Thi-Thuy Le, Okeyo Ally Mwai, Mohamed Nawaz Mohamed Ibrahim, Megan Supple, Beth Shapiro (D), Olivier Hanotte, Guojie Zhang (D), Greger Larson (D), Jian-Lin Han (D), Dong-Dong Wu (D) and Ya-Ping Zhang (D)

Cell Research (2020) 30:824-825; https://doi.org/10.1038/s41422-020-0380-z

Correction to: Cell Research https://doi.org/10.1038/s41422-0200349-y, published online 25 June 2020

We apologize for a mistake in Fig. 2 that was immediately identified by Edward McClure in our article published online on June 25, 2020. In the labels of Fig. 2a, b, the blue color should represent West Asian chickens while the brown color should depict South Asian chickens. The correct version of Fig. 2 is shown below. This correction does not affect the description and conclusion of this article. 
a

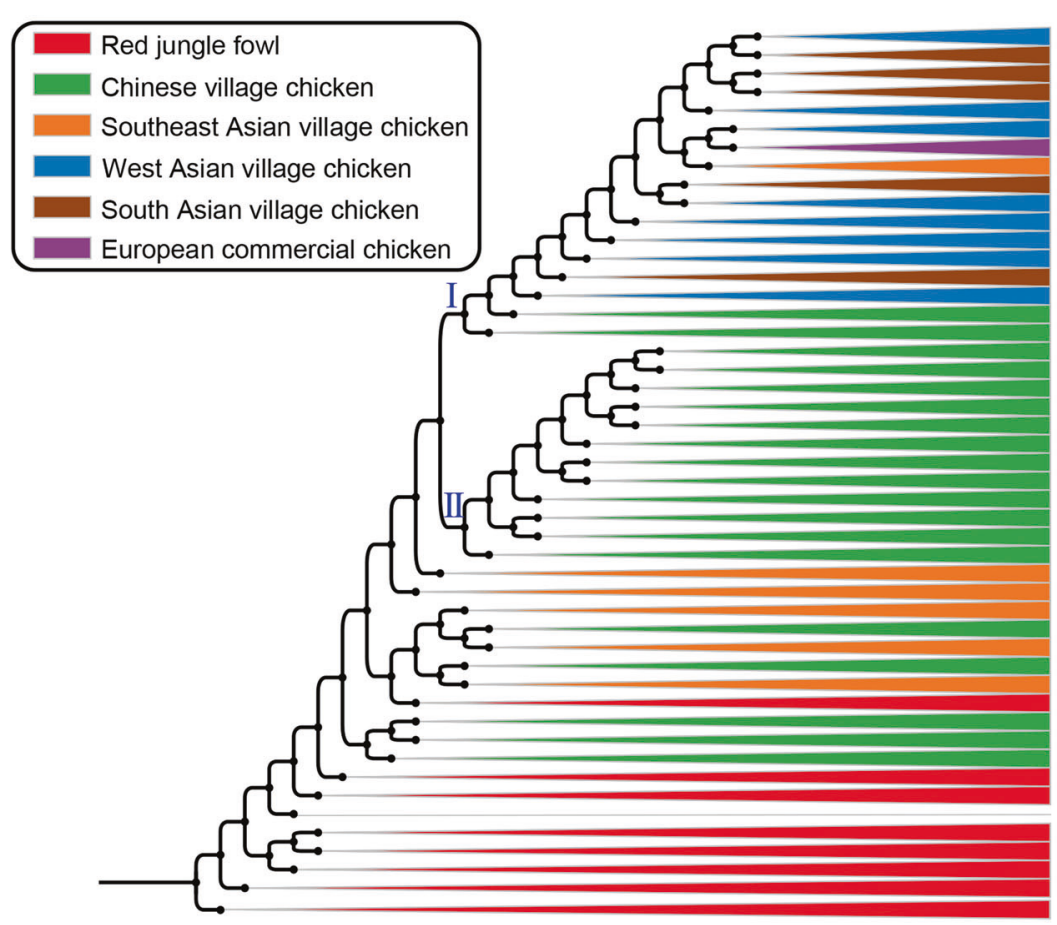

Iran (41); Pakistan (3)

Pakistan (14)

Bangladesh (12); India (6); G. g. spadiceus (3)

Iran (20)

White leghorn (12); India (1); Tibet, China (6)

Indonesia (8); India (2)

Bangladesh (3); Pakistan (13)

Iran (8)

$\operatorname{Iran}(25)$

Iran (3)

Sri Lanka (7); India (2)

Iran (66); India (5); European breeds (15)

Xinjlang,China (14)

Tibet,China (87)

Shand

Henan, China (8)

Liaoning China (8)

Shanxi,China (8)

Jiangxi,China (10)

Jiangxi,China (20)

Peking,China (11)

Tibet, China (4)

Shandong (9) and Xinjiang (10), China

Guangdong,China (7)

Indonesia (2)
Indonesia (11)

Indonesia (14)

Yunnan,China (14)

Yuland (4), Indonesia (1); Vietnam (1); Yunnan (9)

Yunnan, Chi (9); Vietnam (1)

G. g. spadiceus (Thailand X) Xinjiang (1),China

Yunnan (24) and Guangxi (6) China

Tibet,China (15)

Yunnan, China (6)

G. g. spadiceus (32)

Thailand (2)

G. g. murghi (68)

G. g. jabouillei (3)

G. g. jabouillei (24)

G. g. gallus (6)

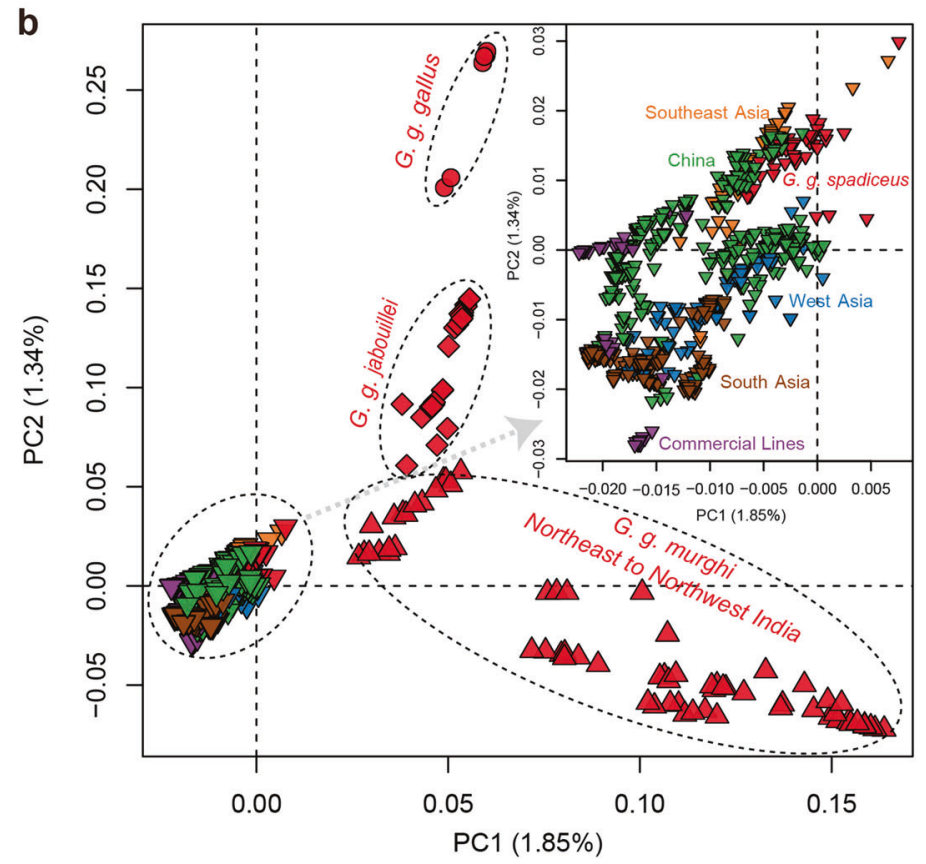

Fig. 2 